\title{
METHOD DEVELOPMENT AND VALIDATION OF DAPAGLIFLOZIN AND SAXAGLIPTIN BY RP-HPLC
}

\author{
Sana Tabassum ${ }^{1}$,Dr.M.Sathishkumar ${ }^{2}$,Dr.A.Mallik ${ }^{3}$, Dr.N.Jyothi ${ }^{4}$ \\ ${ }^{1,2,3,4}$ Department of Pharmaceutical Analysis, Marri Laxman Reddy Institute of Pharmacy, Dundigal, \\ Hyderabad-5000043
}

Article DOI: https://doi.org/10.36713/epra8448

DOI No: 10.36713/epra8448

Corresponding Author: Dr.M.Sathish kumar, V-Block Flat No.102, V-Block Flat No.102, Kompally (village), Telangana

\begin{abstract}
For the coincident evaluation of Dapagliflozin and Saxagliptin in bulk form; Chromatography was run through Intersil-ODS $C_{18}$ column $(250 \mathrm{~mm} \times 4.6 \mathrm{~mm}, 5 \mathrm{micron})$ Mobile phase containing Methanol: Water was pumped through the column in the ratio of 45: 55. The flow rate was $1 \mathrm{ml} / \mathrm{min}$. The temperature help was ambient i.e., upto30 $0^{\circ}$. The optimized selected wavelength was $210 \mathrm{~nm}$. The retention time of Dapagliflozin and Saxagliptin was found to be $4.707 \mathrm{~min}$ and6.68 min respectively. The \% RSD of Dapagliflozin and Saxagliptin was found to be 0.031 and 0.036 respectively. The values of $L O D$ and $L O Q$ obtained from Dapagliflozin and Saxagliptin was $0.56,1.69$ and $0.57,1.74$ respectively. The retention time was decreased and the run time also decreased, so the method development was simple and economical that can be applied successfully for simultaneous estimation of combination of two anti- diabetic drugs; Dapagliflozin and Saxagliptin.

KEY WORDS: Dapagliflozin and Saxagliptin, RP-HPLC.
\end{abstract}

\section{INTRODUCTION}

Method development and validation of Anti-diabetic drugs.i.e., Dapagliflozin and saxagliptin by Reverse phase-HPLC method by using Methanol and Acetonitrile as solvents. Dapagliflozin is an oral diabetics medicine that helps to control blood sugar levels, it helps the kidneys get rid of glucose from your bloodstream. It helps to treat type-2 diabetes along with diet and exercise. Saxagliptin is used as monotherapy or in combination to treat type-2 diabetes.

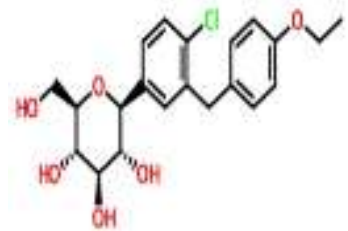

Dapagliflozin

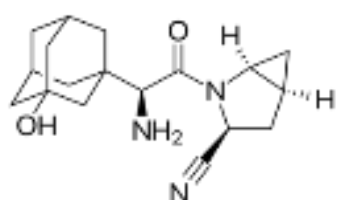

Saxagliptin 


\section{MATERIALS AND METHODS}

Instruments-Instruments

- HPLC -Waters Model NO.2690/5 series Compact System Consisting of Inertsil-C18 ODS column.

- Electronic balance (SARTORIOUS)

- Sonicator( FAST CLEAN)

Substances containing chemicals

- Methanol HPLC Grade.

- Buffer(KH2PO4)Hplc Grade.

Raw Equipment(Unprocessed Materials)

Dapagliflozin and Saxagliptin are working standards.

\section{RESULTS AND DISCUSSION.}

Method development: Method development was done by changing various, mobile phase ratios, buffers et

\section{ADVANCED METHOD (OPTIMIZED METHOD)}

Mobile Phase: Degassed Methanol and Buffer in the ratio of 45:55 V/V.

Preparation of pH 3.4 Phosphate buffer: $2.7218 \mathrm{~g}$ of KH2PO4 was weighed and transferred into a $1000 \mathrm{ml}$ beaker, later it was dissolved and diluted to $1000 \mathrm{ml}$ with HPLC water and the $\mathrm{pH}$ was adjusted to 3.4 with orthophosphoric acid.

Chromatographic conditions that have been optimized

\begin{tabular}{|c|c|}
\hline Parameters & Method \\
\hline Stationary phase (column) & Inertsil -ODS $C_{18}(250$ x $4.6 \mathrm{~mm}, 5 \mu)$ \\
\hline Mobile Phase & Methanol : Buffer (45:55) \\
\hline Flow rate $(\mathrm{ml} / \mathrm{min})$ & $1.0 \mathrm{ml} / \mathrm{min}$ \\
\hline Run time (minutes) & $12 \mathrm{~min}$ \\
\hline Column temperature $\left({ }^{\circ} \mathrm{C}\right)$ & Ambient \\
\hline Volume of injection loop $(\mu \mathrm{l})$ & 20 \\
\hline Detection wavelength (nm) & $210 \mathrm{~nm}$ \\
\hline Drug RT (min) & $\begin{array}{l}\text { 4.707min for Dapagliflozin and } 6.684 \text { for } \\
\text { Saxagliptin. }\end{array}$ \\
\hline
\end{tabular}

Standard chromatogram

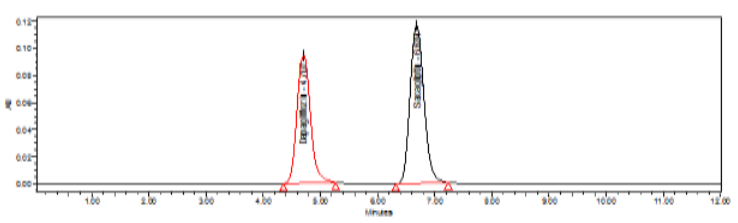

Standard chromatogram

Inference: At RTs of 4.707 minutes for Dapagliflozin and 6.684 minutes for Saxagliptin, a chromatogram was obtained. 
EPRA International Journal of Research and Development (IJRD)

Volume: 6 | Issue: 9 | September 2021

- Peer Reviewed Journal

\begin{tabular}{|c|c|c|}
\hline S.NO & Name of the peak & Retention time(min) \\
\hline 1 & Dapagliflozin & 4.707 \\
\hline 2 & Saxagliptin & 6.684 \\
\hline
\end{tabular}

INFORMATION OF HIGH VALUE (VALIDATION DATA)

PRODUCTS FOR THE SYSTEM (SYSTEM SUITABILITY)

TABLE- 1(a): Data on Dapagliflozin System Suitability

\begin{tabular}{|c|c|c|c|c|}
\hline Injection & RT & Peak Area & USP Plate count & USP Tailing \\
\hline 1 & 4.706 & 674753 & 10953.609752 & 1.153539 \\
\hline 2 & 4.707 & 674261 & 10951.014286 & 1.155271 \\
\hline Mean & 4.7078 & 678433.8 & 10768.34 & 1.155774 \\
\hline SD & 0.001483 & 6031.135 & ----- & ----- \\
\hline \% RSD & 0.031506 & 0.888979 & ------ & ------ \\
\hline
\end{tabular}

TABLE-1(b): Data on Saxagliptin System Suitability

\begin{tabular}{|c|c|c|c|c|}
\hline Injection & RT & Peak Area & USP Plate count & USP Tailing \\
\hline 1 & 6.681 & 1218805 & 9478.317159 & 0.899633 \\
\hline 2 & 6.680 & 1214014 & 9452.196217 & 0.893423 \\
\hline Mean & 6.6826 & 1228593 & 9573.997 & 0.892407 \\
\hline SD & 0.002408 & 122124.07 & ----- & ------ \\
\hline \% RSD & 0.036039 & 1.800764 & ------ & ------ \\
\hline
\end{tabular}

System suitability chromatograms (standards)

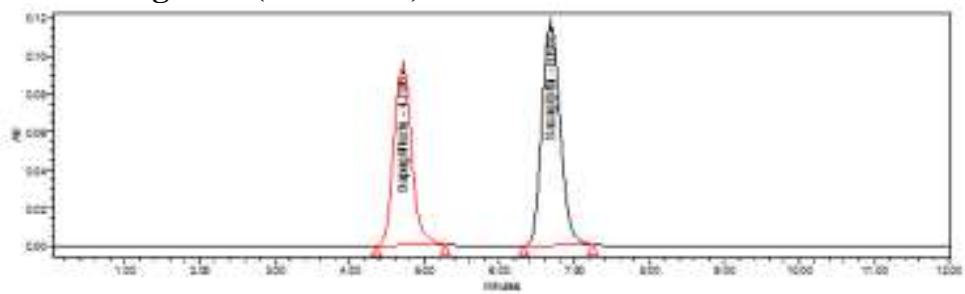

Inference: Standard Chromatogram-1 System Suitability

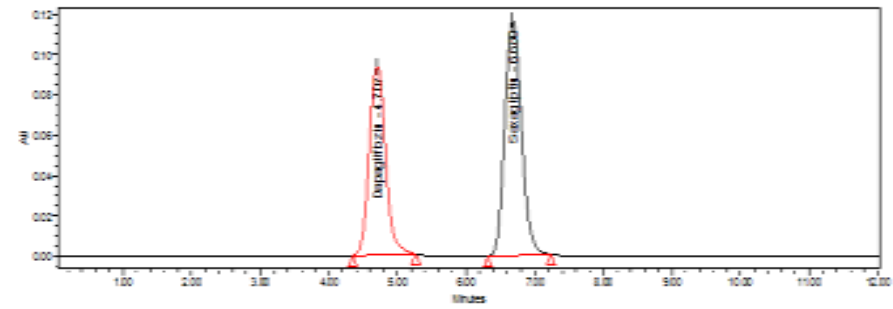

Inference: Norm Chromatogram-2 device appropriateness

DESCRIPTION(SPECIFICITY):

Blank Chromatograph

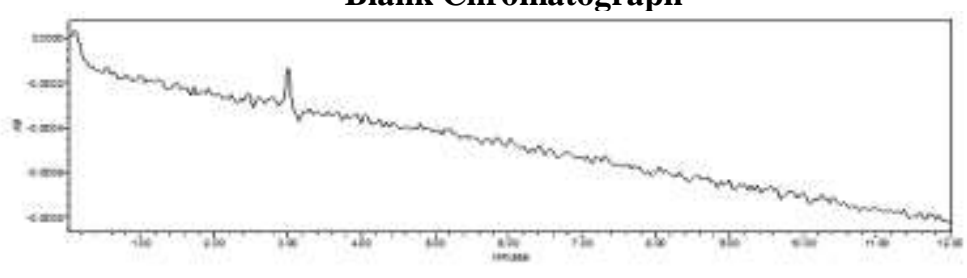

(c) 2021 EPRA IJRD | Journal DOI: https://doi.org/10.36713/epra2016 | www.eprajournals.com |136 | 
Chromatogram Standard

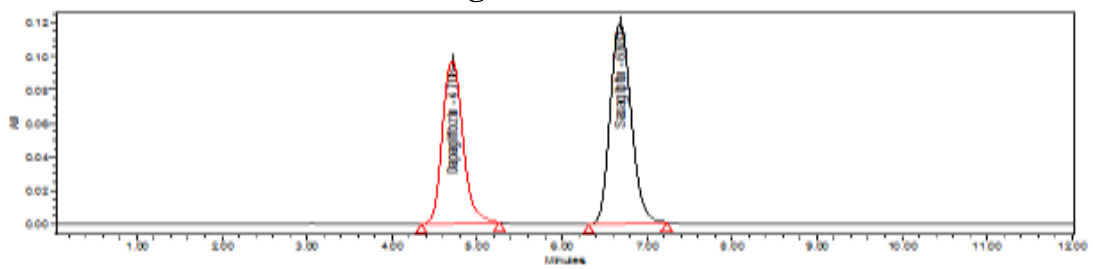

Inference: For Dapagliflozin, a Rt of 4.708min was obtained, while for Saxagliptin, a Rt of 6.682min was obtained.

TABLE-2(i): Data of Repeatability (System precision) forDapagliflozin

\begin{tabular}{|c|c|c|c|}
\hline \multirow{3}{*}{$\begin{array}{c}\text { Concentration } \\
\text { 100ppm }\end{array}$} & Injection & Peak Areas of Dapagliflozin & \%Assay \\
\cline { 2 - 4 } & 1 & 674753 & 98.66 \\
\cline { 2 - 4 } & 2 & 674261 & 99.30 \\
\hline StatisticalAnalysis & Mean & 678433.8 & 100.00 \\
\cline { 2 - 4 } & SD & 6031.135 & 1.107678 \\
\cline { 2 - 4 } & \% RSD & 0.888979 & 1.10 \\
\hline
\end{tabular}

TABLE-2(ii): Information on Saxagliptin Reliability (System Precise)

\begin{tabular}{|c|c|c|c|}
\hline \multirow{3}{*}{$\begin{array}{c}\text { Concentration } \\
\text { 100ppm }\end{array}$} & Injection & Peak Areas of Saxagliptin & \%Assay \\
\cline { 2 - 4 } & 1 & 1218805 & 99.95 \\
\cline { 2 - 4 } & 2 & 1214014 & 100.24 \\
\hline \multirow{3}{*}{ StatisticalAnalysis } & Mean & 1228593 & 99.91 \\
\cline { 2 - 4 } & SD & 22124.07 & 0.35819 \\
\cline { 2 - 4 } & \% RSD & 1.800764 & 0.35 \\
\hline
\end{tabular}

Detailed chromatograms of systems

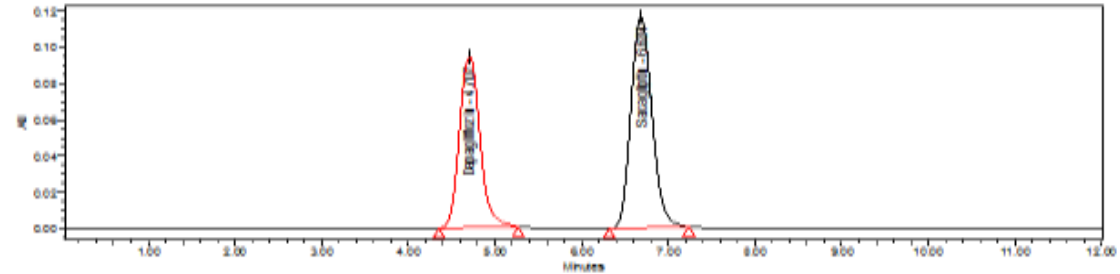

(standard-1)

(b)Method precision

TABLE-3(i): Data of Repeatability (Method precision) for Dapagliflozin

\begin{tabular}{|c|c|c|c|}
\hline $\begin{array}{c}\text { Concentration } \\
\text { 100ppm }\end{array}$ & Injection & Peak Areas of Dapagliflozin & \%Assay \\
\cline { 2 - 4 } & 1 & 633495 & 98.55 \\
\cline { 2 - 4 } & 2 & 635992 & 98.88 \\
\hline \multirow{2}{*}{$\begin{array}{c}\text { Statistical } \\
\text { Analysis }\end{array}$} & Mean & 637312 & 99.278 \\
\cline { 2 - 4 } & SD & 5988.879 & 0.827236 \\
\cline { 2 - 4 } & \% RSD & 0.0891 & 0.83 \\
\hline
\end{tabular}


SJIF Impact Factor 2021: 8.013| ISI I.F.Value:1.241| Journal DOI: 10.36713/epra2016

ISSN: 2455-7838(Online)

EPRA International Journal of Research and Development (IJRD)

Volume: 6 | Issue: 9 | September 2021

- Peer Reviewed Journal

TABLE-3(ii): Data of Repeatability (Method precision) for Saxagliptin

\begin{tabular}{|c|c|c|c|}
\hline $\begin{array}{c}\text { Concentration } \\
\text { 100ppm }\end{array}$ & Injection & Peak Areas of Saxagliptin & \% Assay \\
\cline { 2 - 4 } & 1 & 1202110 & 98.6 \\
\cline { 2 - 4 } & 2 & 1203700 & 99.02 \\
\hline \multirow{2}{*}{$\begin{array}{c}\text { Statistical } \\
\text { Analysis }\end{array}$} & Mean & 1202687.6 & 98.48 \\
\cline { 2 - 4 } & SD & 771.5483 & 0.352647 \\
\cline { 2 - 4 } & \% RSD & 0.1358 & 0.35 \\
\hline
\end{tabular}

Repeatability chromosomes (Repeatable Chromatograms)

Inference: Chromatograph with high repeatability

(Standard-1)

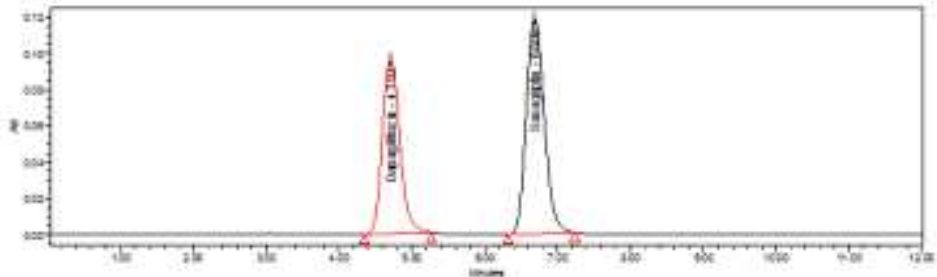

Intermediate precision

Table4: Data of Intermediate precision (Analyst 2) for Dapagliflozin

\begin{tabular}{|c|c|c|c|}
\hline \multirow{2}{*}{$\begin{array}{c}\text { Concentration } \\
\text { 100ppm }\end{array}$} & Injection & Peak Areas of Dapagliflozin & \% Assay \\
\cline { 2 - 4 } & 1 & 636792 & 99.99 \\
\cline { 2 - 4 } & 2 & 634360 & 99.66 \\
\hline \multirow{2}{*}{$\begin{array}{c}\text { Statistical } \\
\text { Analysis }\end{array}$} & Mean & 644607.8 & 0.753536 \\
\cline { 2 - 4 } & SD & 6392.59 & 0.75 \\
\cline { 2 - 4 }
\end{tabular}

(ii)Specifications for Saxagliptin Intermediate (Analyst 2)

\begin{tabular}{|c|c|c|c|}
\hline \multirow{2}{*}{$\begin{array}{c}\text { Concentration } \\
\text { 100ppm }\end{array}$} & Injection & Peak Areas of Saxagliptin & \% Assay \\
\cline { 2 - 4 } & 1 & 1205267 & 99.78 \\
\cline { 2 - 4 } & 2 & 1205625 & 99.95 \\
\cline { 2 - 4 } & 3 & 1205840 & 100.00 \\
\hline \multirow{2}{*}{$\begin{array}{c}\text { Statistical } \\
\text { Analysis }\end{array}$} & Mean & 1206333.5 & 100.19 \\
\cline { 2 - 4 } & SD & 12572.599 & 1.100898 \\
\cline { 2 - 4 } & \% RSD & 1.24 & 1.09 \\
\hline
\end{tabular}

Chromatograms of Intermediate Precision:Inference: 1

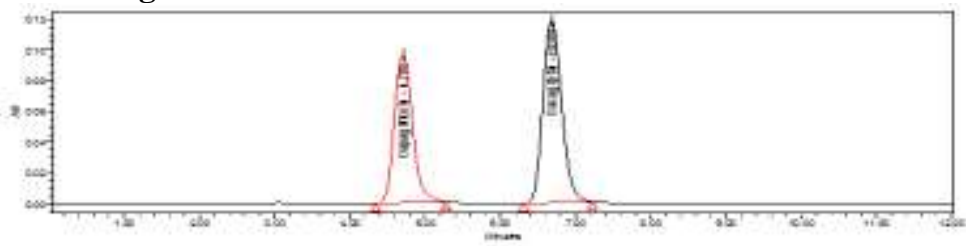

(c) 2021 EPRA IJRD | Journal DOI: https://doi.org/10.36713/epra2016 | www.eprajournals.com |138 | 
Resilience (ACCURACY)

(i)Dapagliflozin data with accuracy

\begin{tabular}{|c|c|c|c|c|c|}
\hline $\begin{array}{c}\text { Concentration } \\
\text { \% of spiked level }\end{array}$ & $\begin{array}{c}\text { Amount } \\
\text { added } \\
\text { (ppm) }\end{array}$ & $\begin{array}{c}\text { Amount } \\
\text { found } \\
\text { (ppm) }\end{array}$ & \% Recovery & \multicolumn{2}{|c|}{$\begin{array}{c}\text { Statistical Analysis of } \\
\text { \% Recovery }\end{array}$} \\
\hline $\begin{array}{c}150 \% \\
\text { Injection 1 }\end{array}$ & 60 & 60.12 & 100.21 & MEAN & 99.97333 \\
\hline $\begin{array}{c}150 \% \\
\text { Injection 2 }\end{array}$ & 60 & 59.76 & 99.61 & & \\
\hline $\begin{array}{c}150 \% \\
\text { Injection 3 }\end{array}$ & 60 & 60.06 & 100.10 & \%RSD & 0.31 \\
\hline
\end{tabular}

(ii)Saxagliptin data with accuracy

\begin{tabular}{|l|l|l|l|l|l|}
\hline $\begin{array}{l}\text { Concentration } \\
\% \text { of spiked level }\end{array}$ & $\begin{array}{l}\text { Amount } \\
\text { added } \\
(\mathrm{ppm})\end{array}$ & $\begin{array}{l}\text { Amount } \\
\text { found } \\
(\mathrm{ppm})\end{array}$ & \% Recovery & \multicolumn{2}{|l|}{$\begin{array}{l}\text { Statistical Analysis of } \\
\% \text { Recovery }\end{array}$} \\
\hline $\begin{array}{l}150 \% \\
\text { Injection 1 }\end{array}$ & 60 & 60.08 & 100.14 & MEAN & 100.02 \\
\hline $\begin{array}{l}150 \% \\
\text { Injection 2 }\end{array}$ & 60 & 59.97 & 99.96 & & \\
\hline $\begin{array}{l}150 \% \\
\text { Injection 3 }\end{array}$ & 60 & 59.98 & 99.98 & \%RSD & 0.09 \\
\hline
\end{tabular}

Chromatograms are used to ensure precision (150 per cent)

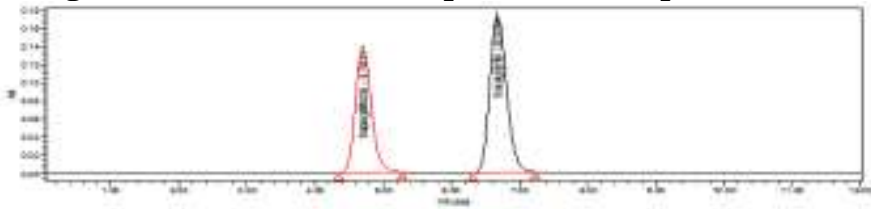

Inference: Standard 1 chromatogram

Variability (LINEARITY):

TABAL 6:Data of Linearity (Dapagliflozin)

\begin{tabular}{|c|c|c|c|}
\hline Concentration (ppm) & Average Area & \multicolumn{2}{|c|}{ Statistical Analysis } \\
\hline 0 & 0 & Slope & 18600 \\
\hline 20 & 632546 & y-Intercept & 276.2 \\
\hline 30 & 658296 & $\begin{array}{c}\text { Correlation } \\
\text { Coefficient }\end{array}$ & 1 \\
\hline 40 & 694400 & & \\
\hline 50 & 730308 & & \\
\hline 60 & 916282 & & \\
\hline 70 & 9402046 & & \\
\hline
\end{tabular}


(a) Dapagliflozin's Linearity Plot (concentration vs response)

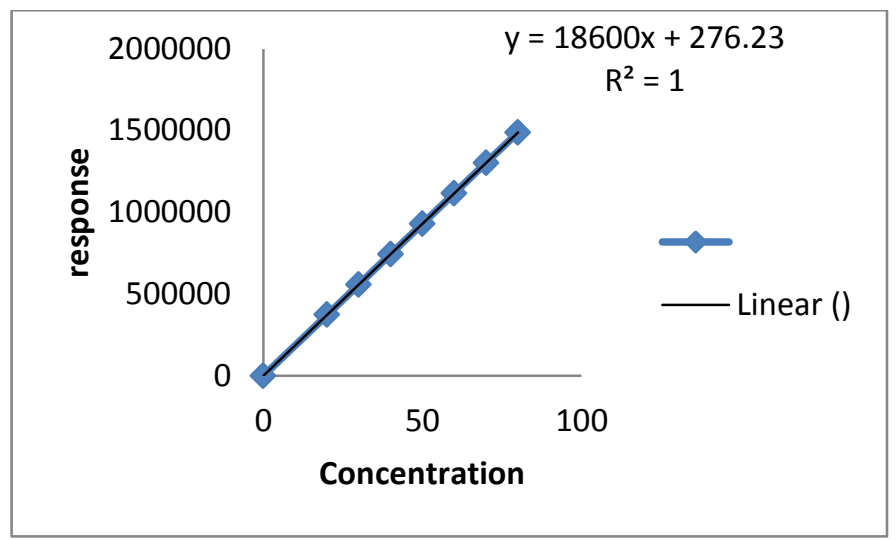

Ii) Details on linearity (Saxagliptin),

\begin{tabular}{|c|c|c|c|}
\hline $\begin{array}{c}\text { Concentration } \\
(\mathbf{p p m})\end{array}$ & $\begin{array}{c}\text { Average } \\
\text { Area }\end{array}$ & \multicolumn{2}{|c|}{ Statistical Analysis } \\
\hline 0 & 0 & Slope & 5140 \\
\hline 20 & 1202965 & y-Intercept & 114.7 \\
\hline 30 & 1254371 & Correlation Coefficient & 1 \\
\hline 40 & 1295856 & & \\
\hline 50 & 1297167 & & \\
\hline 60 & 1308577 & & \\
\hline 70 & 1359903 & & \\
\hline
\end{tabular}

(b)Plot of Saxagliptin Linearity (Concentration Vs Answer)

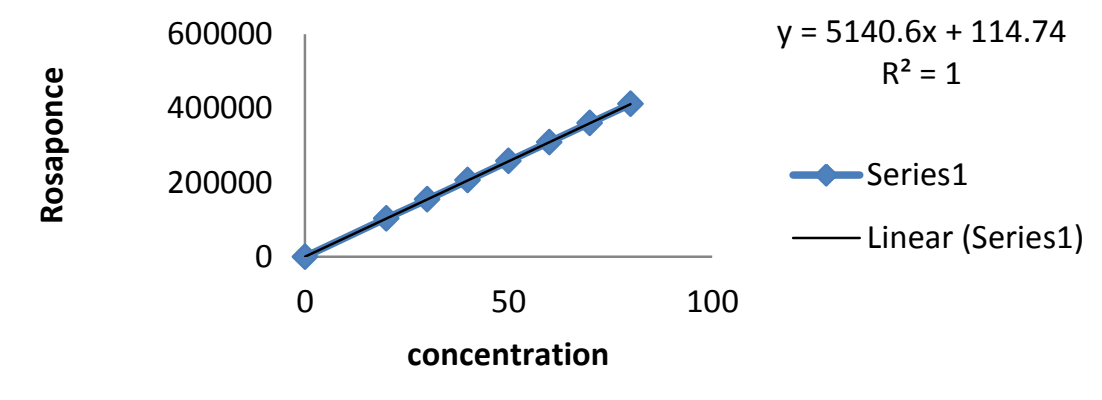

There are chromatograms available. 70 parts per million

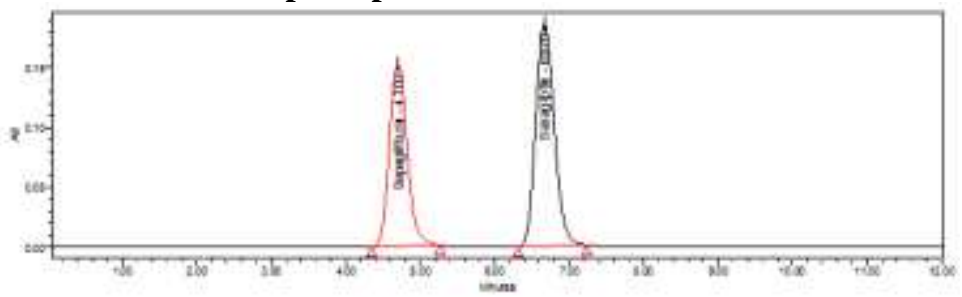

Inference: The standard chromatogram of $70 \mathrm{ppm}$ 
EPRA International Journal of Research and Development (IJRD)

Volume: 6 | Issue: 9 | September 2021

- Peer Reviewed Journal

TABLEMENT: 7

(i) Data on System Variability (Dapagliflozin)

(ii) System-2

\begin{tabular}{|c|c|c|}
\hline S.NO: & Peak area & Assay \% of Dapagliflozin \\
\hline 1 & 634360 & 98.65 \\
\hline 2 & 634098 & 98.63 \\
\hline Mean & 634180.8 & 98.64 \\
\hline \%RSD & 0.019 & 0.12 \\
\hline
\end{tabular}

Data on device variations (Saxagliptin)

System-2

\begin{tabular}{|c|c|c|}
\hline S.NO: & Peak area & Assay \% of Saxagliptin \\
\hline 1 & 1203625 & 99.98 \\
\hline 2 & 1202225 & 99.30 \\
\hline Mean & 1203136.3 & 99.07667 \\
\hline \%RSD & 1.35 & 0.56 \\
\hline
\end{tabular}

Fig42-47,System to system variability chromatograms

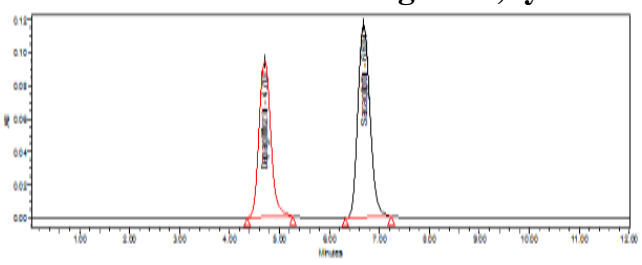

Inference: std- 1

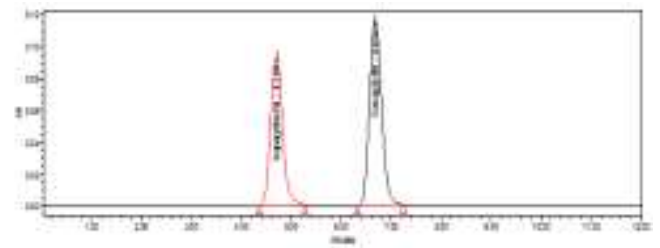

Inference: std- 2

chromatogram showing system-to-system variability

Resiliency (Robustness):

TABLE: 8(i) There's proof that flux rate variability has an impact (Dapagliflozin):

\begin{tabular}{|c|c|c|c|c|c|c|c|c|}
\hline \multirow[t]{6}{*}{$\begin{array}{c}\text { Flow } 0.8 \\
\text { ml }\end{array}$} & Std Area & $\begin{array}{l}\text { Tailing } \\
\text { factor }\end{array}$ & \multirow[t]{6}{*}{$\begin{array}{c}\text { Flow } 1.0 \\
\text { ml }\end{array}$} & $\begin{array}{c}\text { Std } \\
\text { Area } \\
\end{array}$ & $\begin{array}{l}\text { Tailing } \\
\text { factor }\end{array}$ & \multirow[t]{6}{*}{$\begin{array}{c}\text { Flow } 1.2 \\
\mathrm{ml}\end{array}$} & $\begin{array}{c}\text { Std } \\
\text { Area } \\
\end{array}$ & $\begin{array}{l}\text { Tailing } \\
\text { factor }\end{array}$ \\
\hline & 620286 & 1.322089 & & 634322 & 1.604878 & & 602077 & 1.285372 \\
\hline & 619282 & 1.331920 & & 635792 & 1.584354 & & 601854 & 1.319385 \\
\hline & 621337 & 1.296438 & & 634360 & 1.543805 & & 602403 & 1.292055 \\
\hline & 620456 & 1.315454 & & 635696 & 1.568590 & & 603421 & 1.304561 \\
\hline & 620765 & 1.326551 & & 633147 & 1.559986 & & 602465 & 1.294621 \\
\hline Avg & 620425 & 1.31849 & Avg & 634663.4 & 1.572323 & Avg & 602444 & 1.299199 \\
\hline SD & 754.0018 & 0.013728 & SD & 1100.917 & 0.023367 & SD & 599.8833 & 0.013223 \\
\hline \%RSD & 0.086 & 1.04 & \% RSD & 0.184 & 1.48 & \%RSD & 0.09 & 1.01 \\
\hline
\end{tabular}

TABLE: 8(ii) Flow rate shift impact data (Saxagliptin)

\begin{tabular}{|c|c|c|c|c|c|c|c|c|}
\hline \multirow[t]{6}{*}{$\begin{array}{c}\text { Flow } 0.8 \\
\text { ml }\end{array}$} & Std Area & $\begin{array}{l}\text { Tailing } \\
\text { factor }\end{array}$ & \multirow[t]{6}{*}{$\begin{array}{c}\text { Flow } 1.0 \\
\text { ml }\end{array}$} & Std Area & $\begin{array}{l}\text { Tailing } \\
\text { factor }\end{array}$ & \multirow[t]{6}{*}{$\begin{array}{c}\text { Flow } 1.2 \\
\text { ml }\end{array}$} & $\begin{array}{c}\text { Std } \\
\text { Area }\end{array}$ & $\begin{array}{l}\text { Tailing } \\
\text { factor }\end{array}$ \\
\hline & 1273707 & 1.362089 & & 1206349 & 1.280574 & & 1266195 & 1.285372 \\
\hline & 1273211 & 1.352617 & & 1205267 & 1.279932 & & 1265885 & 1.299385 \\
\hline & 1273948 & 1.376926 & & 1205625 & 1.261721 & & 1266303 & 1.308063 \\
\hline & 1273465 & 1.345752 & & 1205840 & 1.276089 & & 1267243 & 1.274662 \\
\hline & 1273862 & 1.374925 & & 1205735 & 1.250640 & & 1265762 & 1.267630 \\
\hline Avg & 1273638.6 & 1.362462 & Avg & 1205763.2 & 1.269791 & Avg & 166277.6 & 1.287022 \\
\hline SD & 3301.369 & 0.013609 & SD & 392.1635 & 0.01314 & SD & 582.9758 & 0.016786 \\
\hline \%RSD & 1.041 & 0.99 & \%RSD & 0.19 & 1.03 & \%RSD & 0.35 & 1.3 \\
\hline
\end{tabular}


Fig48-49, Robustness chromatograms

a) Variation in flow rate (for $0.8 \mathrm{ml} / \mathrm{min}$ flow) has an effect.

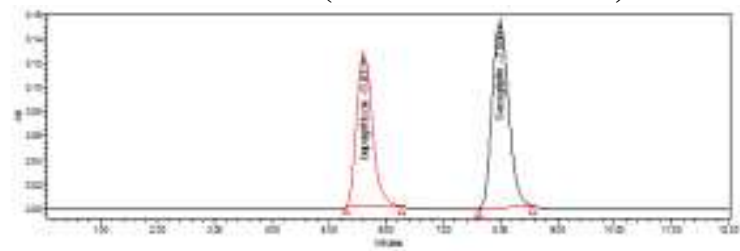

b) Inference: Standard for robustness chromatogram - 1

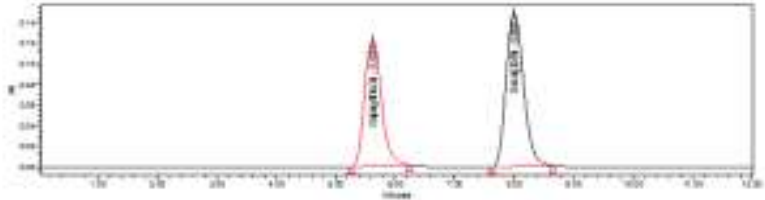

Inference: Standard for robustness chromatogram - 2

SUMMARY AND CONCLUSION

Summary Table

\begin{tabular}{|c|c|c|c|}
\hline Parameters & Dapagliflozin & Saxagliptin & LIMIT \\
\hline Regressioncoefficient & 0.999 & 0.999 & \multirow[t]{4}{*}{$\mathrm{R}<1$} \\
\hline Slope(m) & 18599.8434 & 5140 & \\
\hline Intercept(c) & 276.2281 & 114.73 & \\
\hline $\begin{array}{l}\text { Regression equation } \\
\quad(\mathrm{Y}=\mathrm{mx}+\mathrm{c})\end{array}$ & $\begin{array}{c}\mathrm{y}=18599.8434 \mathrm{x}+ \\
276.2281\end{array}$ & $y=5140 x+114.73$ & \\
\hline Assay (\% mean assay) & $98.64 \%$ & $99.07 \%$ & $90-110 \%$ \\
\hline Specificity & Specific & Specific & $\begin{array}{l}\text { No interference of } \\
\text { any peak }\end{array}$ \\
\hline System precision \% RSD & 0.889 & 1.800 & NMT $2.0 \%$ \\
\hline $\begin{array}{c}\text { Method precision } \\
\text { \% RSD }\end{array}$ & 0.0891 & 0.1358 & NMT $2.0 \%$ \\
\hline Accuracy \% recovery & $99.78 \%$ & $99.80 \%$ & $98-102 \%$ \\
\hline LOD & 0.56 & 1.69 & NMT 3 \\
\hline LOQ & 0.57 & 1.74 & NMT 10 \\
\hline
\end{tabular}

\section{CONCLUSION}

Chromatography was run through Intersil-ODS $\mathrm{C}_{18}$ column $(250 \mathrm{~mm} \times 4.6 \mathrm{~mm}, 5 \mathrm{micron})$ Mobile phase containing Methanol: Water was pumped through the column in the ratio of 45: 55. The flow rate was $1 \mathrm{ml} / \mathrm{min}$. The temperature help was ambient i.e., upto $30^{\circ} \mathrm{c}$. The optimized selected wavelength was $210 \mathrm{~nm}$. The retention time of Dapagliflozin and Saxagliptin was found to be $4.707 \mathrm{~min}$ and6.68 $\mathrm{min}$ respectively. The \%RSD of Dapagliflozin and Saxagliptin was found to be 0.031 and 0.036 respectively. The values of LOD and LOQ obtained from Dapagliflozin and Saxagliptin was 0.56, 1.69 and 0.57, 1.74 respectively. The retention time was decreased and the run time also decreased, so the method development was simple and economical that can be applied successfully for simultaneous estimation of combination of two anti- diabetic drugs; Dapagliflozin and Saxagliptin. 


\section{EPRA International Journal of Research and Development (IJRD)

\section{REFERANCES}

1. Lindholm.J, Development and Validation of HPLC Method for Analytical and Preparative purpose. Acta Universitatis Upsaliensis, pg. 13-14, (2004).

2. Dr.S. Ravi Shankar, Text book of pharmaceutical analysis, Fourth edition, Pg. 13.1-13.2

3. Connors Ka. A Textbook of Pharmaceutical Analysis, Wiley intercedences Inc; Delhi, 3rd Ed, Pg. 373-421, (1994)

4. Gurdeep R.Chatwal, Sham K. Anand, Instrumental Methods of Chemical Analysis 2.566-2.638 (2007)

5. ICH, Validation of analytical procedures: Text and Methodology. International Conference on Harmonization, IFPMA, Geneva, (1996)

6. K. D. Tripathi, Essentials of Medical Pharmacology, 6th Edition, Jaypee brother's medical publishers $(P)$ LTD, p-254-255.

7. Indian Pharmacopoeia, Indian Pharmacopoeia Commission, Controller of Publication, Government of India, Ministry of health and Family Welfare, Ghaziabad, India, 2 (2010) 1657-1658.

8. British Pharmacopoeia, The British Pharmacopoeia Commission, the stationary office, UK, London, 140814092 (2011).

9. https://www.drugbank.ca/drugs/DB00394

10. https://www.drugbank.ca/drugs/DB01001 